\title{
Evaluación de dos fórmulas de yogur enriquecido con harina de Acheta domesticus y harina de Brosimum alicastrum Swartz como alimentos complementarios
}

\author{
Evaluation of two formulas of yogurt enriched with Acheta domesticus flour and Brosimum \\ alicastrum flour, as complementary foods
}

Sergio A. Hernandez*, Claudia I. Ralda, Axel J. Godoy, Edgar A. Polanco, Hugo R. Pérez

Facultad de Medicina Veterinaria y Zootecnia, Universidad de San Carlos de Guatemala, Guatemala

*Autor al que se dirige la correspondencia: hernandez_sergio@usac.edu.gt

Recibido: 29 de noviembre 2018 / Revisión: 17 de mayo 2019 / Aceptado: 21 de junio 2019

\section{Resumen}

$\mathrm{L}$ a prevalencia de desnutrición en niños en Guatemala es constante y se ha mantenido alta por años. Tomando en cuenta esta problemática se planteó un modelo de alimentación complementaria a base de yogur enriquecido con harinas de alto valor proteico en niños de edad preescolar en el suroccidente de Guatemala. Los tratamientos evaluados fueron yogur con harina de Acheta domesticus (grillo), yogur con harina de Brosimum alicastrum Swartz (ramón) y un grupo control. Se evaluó el peso, talla y velocidad de crecimiento durante cuatro meses brindando $125 \mathrm{~mL}$ de yogur cinco días por semana. La ganancia media (desviación estándar) de peso en kg fue de 3.21 (1.84), 2.22 (1.14) y 1.67 (0.64) para A. domesticus, B. alicastrum y grupo control, respectivamente. Mostrando la mezcla de yogur con harina de $A$. domesticus diferencia significativa frente al control $(p<.05)$, por el contrario, no se encontró diferencia significativa $(p>.05)$ cuando se comparó con yogur con harina de $B$. alicastrum. Para el incremento en talla en cm los resultados fueron 4.61 (1.13), 4.25 (1.54) y 3.07 (1.84) para A. domesticus, B. alicastrum y grupo control, respectivamente, mostrando ambas diferencias significativas frente al control $(p<.05)$. Se determinaron los costos por dosis los cuales fueron US\$0.56 (A. domesticus) y US\$0.36 (B. alicastrum). Se concluye que el yogur con harina de $A$. domesticus fue el que presentó mejor respuesta en las variables de estudio.

Palabras claves: harina de grillo, harina de ramón, desnutrición, alimentos funcionales

\begin{abstract}
$\mathrm{T}$ The prevalence data on malnutrition of children in Guatemala are constant. Taking into account this problem, a yogurt-based supplementary feeding model was proposed, enriched with high-protein flours in pre-school children in southwestern Guatemala. The treatments evaluated were yogurt with Acheta domesticus flour (cricket), yogurt with Brosimum alicastrum Swartz flour (ramon) and control group. The weight, height and growth rate were evaluated during four months providing $125 \mathrm{~mL}$ of yogurt five days per week. The weight gain in $\mathrm{kg}$ was 3.21 (1.84), 2.22 (1.14) and 1.67 (0.64) for A. domesticus, B. alicastrum and control group respectively, showing significant difference $(p<.05)$. For the increase in height in $\mathrm{cm}$, the results were 4.61 (1.13), 4.25 (1.54) and 3.07 (1.84) for A. domesticus, B. alicastrum and control group respectively, showing significant difference $(p<.05)$. The costs per dose were determined, which were US $\$ 0.56$ (A. domesticus) and US $\$ 0.36$ (B. alicastrum). The study concludes that yogurt with $A$. domesticus flour was the one that presented the best response in the study variables.
\end{abstract}

Keywords: cricket flour, ramon flour, malnutrition, functional foods 


\section{Introducción}

En Guatemala el acceso a los alimentos de alto valor nutricional en el área rural sigue siendo una limitante para el adecuado crecimiento infantil. La evolución de la desnutrición crónica desde 1987 hasta 2015 ha variado de $62.2 \%$ a $46.5 \%$ (Secretaría de Seguridad Alimentaria y Nutricional [Sesan], 2015). Estos datos indican una mínima reducción de $0.57 \%$. Está lenta reducción se suma a factores como falta de empleo, ausencia de servicios básicos, inseguridad, migración, abandono, entre otros que limitan la mejora en peso y talla de casi la mitad de niños en edad preescolar. Actualmente, la mayoría de las acciones encaminadas a la reducción de la desnutrición se enfocan en niños de edades tempranas, desde la atención a madres gestantes, nacimiento, hasta los 6 años.

Estos programas de alimentación escolar son variables dependiendo de la disponibilidad de productos, la solvencia económica de la organización de padres de familia quienes reciben un aporte estatal. Sin embargo, los menús recomendados por el Ministerio de Educación contemplan pocas fuentes de proteína de origen animal lo cual repercute en el desarrollo neurológico y futuro desarrollo cognoscitivo de los niños (Sesan, 2015). Según datos de un estudio elaborado por la Organización de las Naciones Unidas para la Alimentación y la Agricultura (FAO) en 2013, el actual programa de alimentación escolar tiene una cobertura del $85.3 \%$ de las escuelas distribuidas a nivel nacional.

Las edades de 0-6 años son una etapa crucial para el desarrollo de la persona, puesto que una mala alimentación puede provocar un impacto irremediable, que tendrá efectos adversos a lo largo de toda la vida de la persona (Victora et al., 2008). En este sentido, el uso de alimentos funcionales en este segmento vulnerable de la población rural supone una opción real para mitigar el efecto de la desnutrición crónica. Un alimento funcional tiene el potencial de convertirse en potenciador del desarrollo y la diferenciación en el organismo, actúan como moduladores del metabolismo de nutrientes, la expresión génica, el estrés oxidativo y la esfera psíquica (Silveira, Monereo, \& Molina, 2003).

El uso de yogur de leche bovina se propone en este estudio debido a que es una fuente excelente de alimento que ha sido utilizada en múltiples proyectos para alimentación de niños. Varios estudios han obtenido resultados satisfactorios en cuanto a la aceptación del producto, así como la mejora del estado nutricional de los beneficiarios de estos proyectos (Ponce, 2008).
Además de ello, las guías alimentarias de Guatemala establecen que el yogur y otros productos lácteos son un alimento básico para el ser humano, es por ello que la utilizan en la dieta escolar (Ministerio de Salud Pública y Asistencia Social [MSPAS], 2012).

En el 2017 se creó la Ley de Refacción Escolar la cual establece que se deberá asignar un rubro específico de como mínimo cuatro quetzales (US\$0.52) por beneficiario. Esto demuestra que el modelo de alimentación complementaria propuesto puede ser incluido dentro de la refacción escolar estipulada en el proyecto de ley de alimentación escolar 16-2017 en el Decreto del Congreso de la República (2017).

La harina de Acheta domesticus (Grillo) posee elementos nutricionales como altos niveles de proteína (62\%), aminoácidos, minerales y aceites esenciales, además de la sustentabilidad de su producción por lo que fue incluido en esta investigación. Según la revisión de Apolo-Arévalo y Lannacone (2015) los grillos contienen un alto valor proteico en comparación con otros productos de origen animal además de ser viable su producción en condiciones sostenibles. Otro estudio realizado por Nakagaki Sunde y Defoliart (1986) demostró que el grillo doméstico es una fuente de proteína y aminoácidos esenciales y su uso puede ser una alternativa dependiendo del costo y viabilidad de producción. Otro aspecto a favor del potencial para la crianza de grillos es reportado por Nakagaki y Defoliart (1991) quienes indicaron una mejor conversión y eficiencia alimenticia de estos insectos en comparación con otras especies como pollos de engorde y cerdos. En relación a la aceptación de productos con inclusión de harina de grillo Zhong (2017) reportó en un estudio donde elaboró una barra nutritiva que fue aceptada parcialmente por los consumidores en comparación con barras disponibles en el mercado, el autor señala que deben de mejorarse los atributos sensoriales de los alimentos con dicha harina.

La semilla del árbol de Brosimum alicastrum Swartz (ramón) por su contenido de proteína (9\%), energía (350 kcal/100 g), aceites esenciales, grasas, azucares y aminoácidos esenciales ha sido utilizada en programas de ayuda a mujeres para encontrar medios de subsistencia, mejorar la salud y nutrición de sus familias, así como para obtener ingresos para el hogar. Con la semilla de ramón se puede producir harina negra que se utiliza para la fabricación de pan o un tipo de tortilla. Esta especie posee amplia distribución en la región mesoamericana y existen reportes de uso por parte de la cultura maya. Algunos estudios recopilados 
por Lentz, Dunning y Scarborough (2015) este árbol es predominante en los bosques de altura y en transición, lo que lo ubica como una planta multipropósito. De acuerdo a Carter (2015) la harina de semilla de ramón puede ser utilizada como sustituto de la harina de trigo en formulaciones para la elaboración de galletas, el único hallazgo fue la coloración final del producto con mayor inclusión de harina el cual mostró un color mezclado entre marrón y amarillo. Otro estudio efectuado en Guatemala por Martínez (2014) donde se evaluó el uso de harina de la semilla de ramón como parte de la formulación de un atol (bebida) encontró que luego de evaluar diferentes proporciones de harina de ramón y maíz, la prueba de preferencia indicó la mezcla 75:25 fue la mayormente aceptada por los consumidores. Esto muestra que la harina de ramón debidamente incluida en alimentos socialmente aceptados por la población puede proveer de una suplementación proteica al consumidor.

En este estudio se evaluó: (1) la respuesta al consumo de yogur enriquecido con harina de $A$. domesticus y yogur enriquecido con harina de $B$. alicastrum a partir de las variables de ganancia de peso e incremento de talla, (2) la aceptación y preferencia del consumo en niños de 4-6 años y (3) la viabilidad económica del uso de yogur como alternativa de alimentación en términos de costos de producción.

\section{Materiales y métodos}

La investigación se desarrolló bajo un enfoque cuantitativo explicativo con diseño cuasi experimental. El período de estudio fue de 4 meses y se desarrolló en el municipio de Patulul, departamento de Suchitepéquez, Guatemala. El grupo de estudiantes en el estudio fueron matriculados en preescolar de la Escuela Tecún Umán, Patulul, Suchitepéquez. La muestra se seleccionó por conveniencia y contempló 15 niñas y 13 niños que fueron la totalidad de la matrícula estudiantil del grado quedando excluidos tres niños cuyos padres no avalaron su participación. Como grupo control se tomaron los datos proporcionados por el Centro de Salud de cuatro niñas y ocho niños entre 4-6 años del área de Patulul, quienes no consumieron ninguno de los tratamientos evaluados.

El estudio evaluó la utilización de $125 \mathrm{~mL}$ de yogur de leche de vaca con mermelada y adición de harina de $A$. domesticus. El otro tratamiento fue la misma dosis de yogur con mermelada y adición de harina de B. alicastrum. La frecuencia de consumo fue de 5 días a la semana durante 4 meses. Esta dosis fue recomendada por un especialista quien indicó la dosis y la cantidad de harina a incluir. Las guías alimentarias para Guatemala recomiendan un máximo de un vaso $(250 \mathrm{~mL})$ por día de productos lácteos (MSPAS, 2012).

Los productos fueron elaborados en la finca San Julián propiedad de la Facultad de Medicina Veterinaria de la Universidad de San Carlos de Guatemala. El yogur fue elaborado con leche de vaca proveniente de la misma finca, se realizó el monitoreo de buenas prácticas de ordeño y control de mastitis, se utilizó un pasteurizador de $15 \mathrm{lL}$ de capacidad en donde se incubó la leche, luego fue añadido el cultivo liofilizado (Lactobacillus bulgaricus y L. lactis). Posteriormente se extrajo el coágulo del pasteurizador, se colocó en una cámara de enfriamiento para luego de alcanzar los $4{ }^{\circ} \mathrm{C}$ se adicionó un litro de mermelada y $480 \mathrm{~g}$ de harina de $A$. domestica y para el otro tratamiento 480 $\mathrm{g}$ de harina de $B$. alicastrum. La mermelada utilizada fue de fresa y fue elaborada en la finca a razón de tres medidas de fruta, una de agua y una de azúcar. Las harina de $A$. domestica fue adquirida con un productor, Zoot Hersson Giovanny Icu Cutzal del área de Chimaltenango, Guatemala (herssonicu@hotmail.com) y la harina de B. alicastrum fue adquirida con Comunidad La Bendición San Miguel Pochuta, Chimaltenango (Codemur, codemurguate@yahoo.com) proveedores especializados en su elaboración. Se tomaron muestras periódicamente para asegurar la calidad microbiológica de los productos suministrados en el estudio. También se realizaron estudios en el Laboratorio de Bromatología de la Facultad de Medicina Veterinaria y Zootecnia de los productos elaborados con la finalidad de obtener información sobre el valor nutritivo de los tratamientos.

Para integrar la muestra de niños en el estudio se realizó una charla informativa con los padres de familia para dar a conocer las especificaciones del estudio, y posteriormente firmaron un consentimiento informado que contempló estándares internacionales de bioética. Los padres quedaron en libertad de permitir a sus hijos participar en el estudio. La distribución de la muestra fue dividida en dos grupos completamente al azar los cuales conformaron los dos tratamientos. Durante la fase experimental se realizó una encuesta $(n=21)$ con los padres de los niños, para caracterizar la frecuencia y tipos de alimentos a los que los niños tuvieron acceso durante el estudio.

Las mediciones antropométricas se realizaron de acuerdo a técnicas estandarizadas (Ghisays et al., 2018; Britz, Aznarez, \& Della Santa, 2017). Todas las mediciones fueron realizadas por el mismo técnico capacita- 
do. Las medidas se tomaron con la menor ropa posible y cualquier objeto que pudiera alterar el peso, luego se indicó a la niña o niño colocarse de pie en una postura adecuada. Al inicio del estudio se registró el peso y talla de los niños en una hoja electrónica de Microsoft Excel ${ }^{\circledR}$ posteriormente se realizó un registro mensual de peso y talla utilizando una balanza que incluía tallimetro con sistema de brazo y pilones debidamente calibrada. De igual forma se monitoreo el estado de salud de los niños que estuvieron suplementados con la supervisión del Centro de Salud de Patulul, Suchitepéquez. Los costos se registraron de igual forma para determinar el costo de producción de una dosis de cada tratamiento.

La evaluación de aceptación y preferencia de los tratamientos fue realizada mediante una prueba sensorial previo al inicio de la fase experimental. Se utilizó una boleta para evaluar la aceptación de las características de color, olor, sabor y textura de cada tratamiento evaluado. Durante la evaluación se brindó acompañamiento por parte de personal capacitado. Esta prueba contempló una escala simbólica representada por figuras con expresión de agrado y desagrado que fueran fácilmente identificadas por los niños. Posteriormente se realizó la prueba de preferencia en la que se pidió que seleccionaran el tratamiento que fue de mayor agrado.

Para las variables peso y talla se estimaron los valores de respuesta de los tratamientos utilizando un análisis de varianza con una prueba post-hoc de Tukey con un valor de $p<.05$. La variable velocidad de crecimiento fue analizada por medio de estadística descriptiva. Para determinar la aceptación se utilizó una prueba exacta de Fisher con valor de $p<.05$ en cuanto a la preferencia se utilizó estadística descriptiva. La estimación de costos se realizó por medio del método de costeo variable con el que se establecieron los costos de producción por dosis de $125 \mathrm{~mL}$ de cada tratamiento.

Para el análisis estadístico se utilizó un formulario procesado en una hoja electrónica de Microsoft Excel ${ }^{\circledR}$ y los datos recabados mensualmente se procesaron utilizando los softwares MegaStat2007® Versión 10.1 y PAST ${ }^{\circledR}$ Versión $2.17 \mathrm{c}$. Para el análisis de costos de producción ser utilizó una base de datos en Microsoft Excel ${ }^{\circledR}$, tomando en cuenta los recursos necesarios para elaborar los dos tipos de alimento funcional evaluados.

\section{Resultados}

Se realizó un análisis del contenido nutricional para cada uno de los tratamientos con la finalidad de conocer el aporte de nutrientes de cada alimento. En la Tabla 1 se observan los valores nutricionales del yogur enriquecido con harinas. La Figura 1 muestra los resultados de la caracterización de la frecuencia y tipo de alimentos a los que los niños tuvieron acceso durante el periodo de estudio.

\section{Ganancia de peso}

Para la variable ganancia de peso se determinó que hubo diferencia significativa $(p<.05)$ en el tratamiento de yogur con harina de $A$. domesticus respecto al control, mientras que el tratamiento de yogur con harina de $B$. alicastrum no presentó diferencia significativa (Tabla 2). El análisis de los datos muestra que el peso medio de los niños del tratamiento de yogur con harina de $A$. domesticus fue superior a los otros dos tratamientos. Por otro lado, los pesos de los niños del tratamiento de yogur con $B$. alicastrum no tuvieron diferencia respecto al grupo control.

\section{Incremento de talla}

Respecto a la variable talla el análisis estadístico presentó diferencia significativa $(p<.05)$ por lo que se realizó una prueba post hoc de Tukey evidenciando que ambos tratamientos fueron superiores al control, pero no entre sí (Tabla 2). Se determinó que la mejor respuesta fue la de los niños del tratamiento de yogur con harina de $A$. domesticus, seguido por el tratamiento de yogur con B. alicastrum.

\section{Velocidad de crecimiento}

En el análisis de la velocidad de crecimiento con relación al peso se obtuvo un promedio de $0.80(0.44)$ $\mathrm{kg} / \mathrm{mes}$ durante el periodo de estudio para los niños del tratamiento de yogur con harina de A. domesticus. El promedio (desviación estándar) de aumento en el peso fue de $0.56(0.27) \mathrm{kg} / \mathrm{mes}$ para los niños del tratamiento de yogur con $B$. alicastrum. En la Figura 2 se puede observar el ritmo de ganancia de peso de los dos tratamientos evaluados. . En cuanto al grupo control según datos proporcionados por el Centro de Salud, se determinó una ganancia de peso de $0.28(0.10) \mathrm{kg} / \mathrm{mes}$ en niños que no consumieron ninguno de los tratamientos.

La velocidad de crecimiento respecto a la talla de los niños reportó un aumento de $1.15(0.28) \mathrm{cm} / \mathrm{mes}$ para los niños que consumieron yogur con $A$. domesticus. Por otro lado, los niños que consumieron yogur con 
Tabla 1

Contenido nutricional del yogur enriquecido con harina de $\mathrm{B}$. alicastrum y yogur enriquecido con A. domesticus

\begin{tabular}{|c|c|c|c|c|}
\hline Tratamientos & Extracto etéreo $(\%)$ & Fibra cruda $(\%)$ & Proteína $(\%)$ & Cenizas $(\%)$ \\
\hline $\begin{array}{l}\text { Yogur enriquecido con harina de } A \text {. } \\
\text { domesticus }\end{array}$ & 0.27 & 0.63 & 9.21 & 0.61 \\
\hline $\begin{array}{l}\text { Yogur enriquecido con harina de } B \text {. } \\
\text { alicastrum }\end{array}$ & 0.19 & 1.11 & 12.40 & 0.59 \\
\hline
\end{tabular}

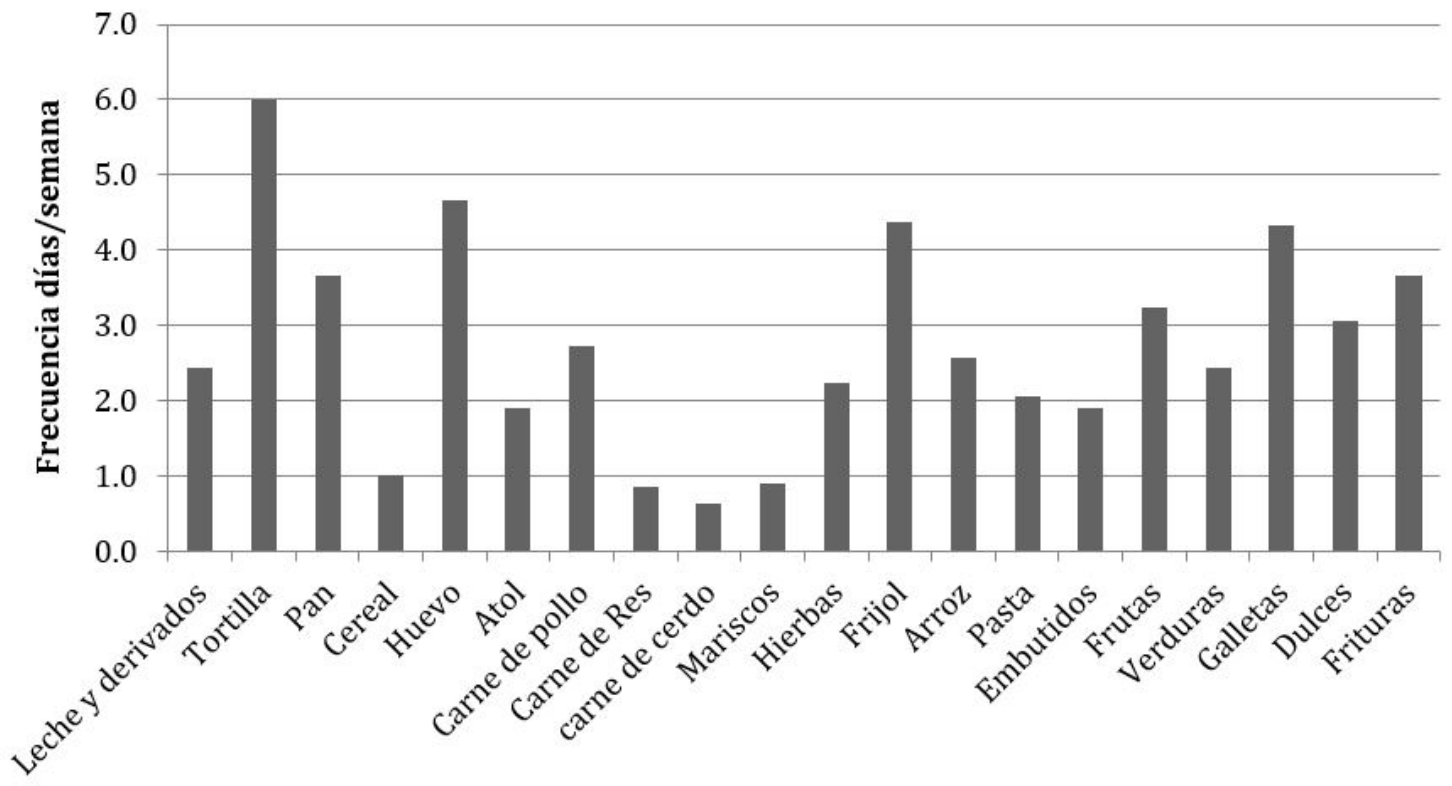

Figura 1. Frecuencia y tipos de alimentos en la dieta de los niños

Tabla 2

Ganancia de peso y talla

\begin{tabular}{lll}
\hline Tratamiento & $\begin{array}{l}\text { Ganancia de peso }(\mathrm{kg}) \\
\text { Media (desviación estándar) }\end{array}$ & $\begin{array}{l}\text { Talla }(\mathrm{cm}) \\
\text { Media (desviación estándar) }\end{array}$ \\
\hline A. domesticus & $3.21(1.84) \mathrm{a}^{*}$ & $4.61(1.13) \mathrm{a}^{*}$ \\
B. alicastrum & $2.22(1.14) \mathrm{ab}^{*}$ & $4.25(1.54) \mathrm{a}^{*}$ \\
Grupo control & $1.67(0.64) \mathrm{b}^{*}$ & $3.07(1.84) \mathrm{b}^{*}$ \\
\hline
\end{tabular}

Nota. $*$ Valores con igual literal no presentan diferencia significativa $(p>.05)$ 


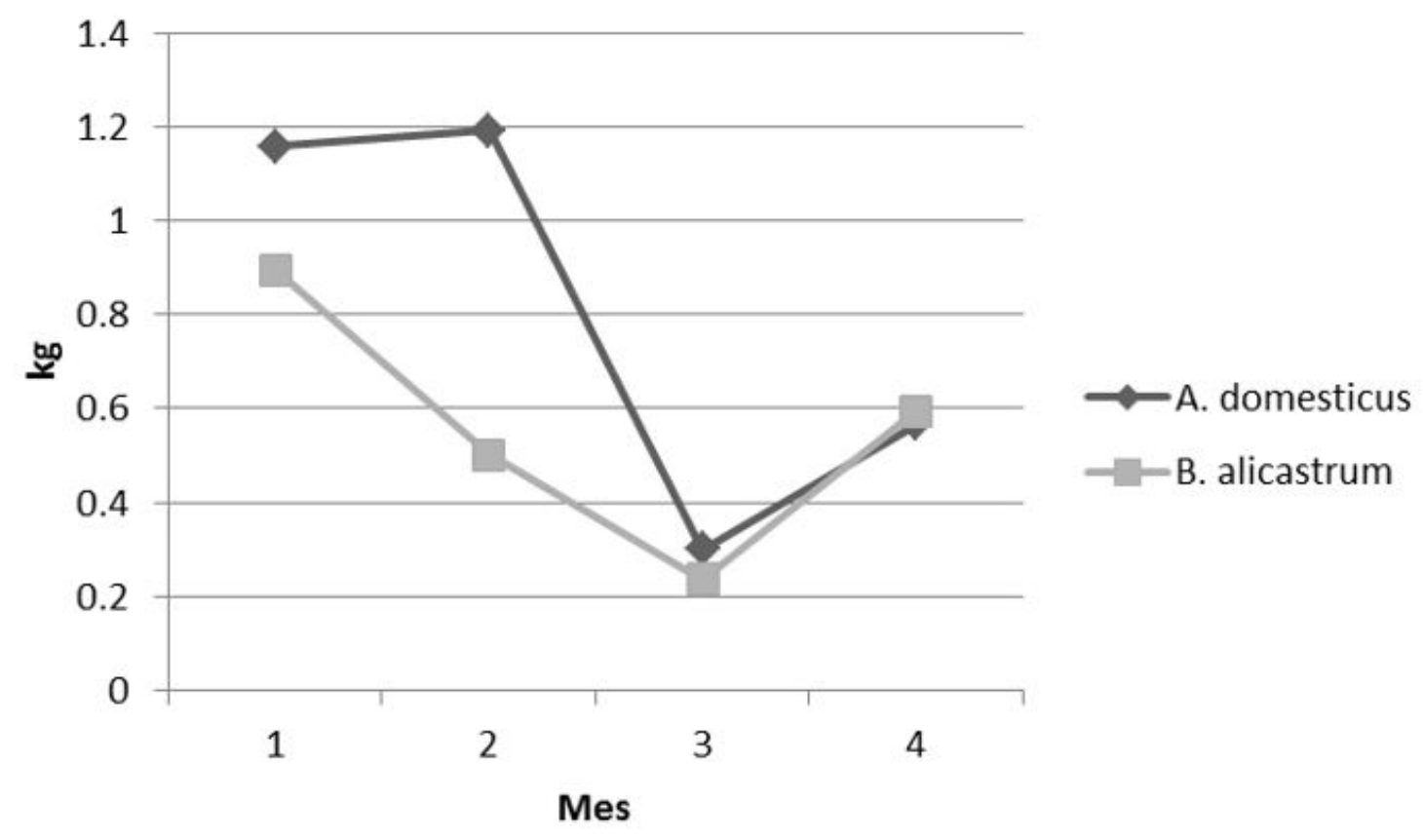

Figura 2. Velocidad de crecimiento en relación al peso

B. alicastrum reportaron un aumento de talla promedio de $1.06(0.38) \mathrm{cm} / \mathrm{mes}$. En la Figura 3 se indica el ritmo de aumento de talla de los niños incluidos en el estudio. En cuanto al grupo control según datos proporcionados por el Centro de Salud, se determinó un aumento de talla de $0.51(0.31) \mathrm{cm} / \mathrm{mes}$ en niños que no consumieron ninguno de los tratamientos.

\section{Prueba de aceptación}

Se evaluó la aceptación de los tratamientos en cuanto a olor, color, sabor y textura. Para las variables olor, color y textura no se encontró diferencia significativa $(p>.05)$. En cuanto al sabor, el tratamiento de yogur con harina de $A$. domesticus fue superior al tratamiento de yogur con harina de B. alicastrum presentando diferencia significativa $(p<.05)$. Se realizó una prueba de preferencia en la cual 29/31 (93.51\%) niños eligieron el yogur enriquecido con harina de $A$. domesticus.

\section{Determinación de costos por dosis}

Se determinaron los costos de producción por porción de $125 \mathrm{~mL}$ de yogur enriquecido con harina de $A$. domesticus y yogur enriquecido con harina de $B$. alicastrum, presentando un costo mayor el primer tratamiento mencionado con $\$ 0.56$ y el segundo tratamiento con $\$ 0.36$.

\section{Discusión}

Se observó que ambos alimentos funcionales de yogur enriquecido con harinas presentan un alto valor nutricional. Estos alimentos son ideales para enriquecer las dietas de los niños en edad preescolar, incluso muestran mayores valores a los presentados por otros autores que utilizaron yogur sin ningún aditivo en cuanto a proteína, fibra, cenizas y extracto etéreo (Barril-Cuadrado, Puchulu, \& Sánchez, 2013; Huertas, 2014). Esto indica que los alimentos elaborados en el presente estudio cumplen con lo recomendado por las dietas de niños preescolares incluidas en las guías alimentarias para Guatemala, las cuales indican que se debe de incluir leche, yogur, cereal, frutas y azúcares (MSPAS, 2012). En la presente investigación se desarrolló una alternativa que podría contribuir a reducir la desnutrición en zonas rurales, ya que según el censo de talla realizado por El Sistema de Información Nacional de Seguridad Alimentaria y Nutricional (SIINSAN) (2015) de 984 


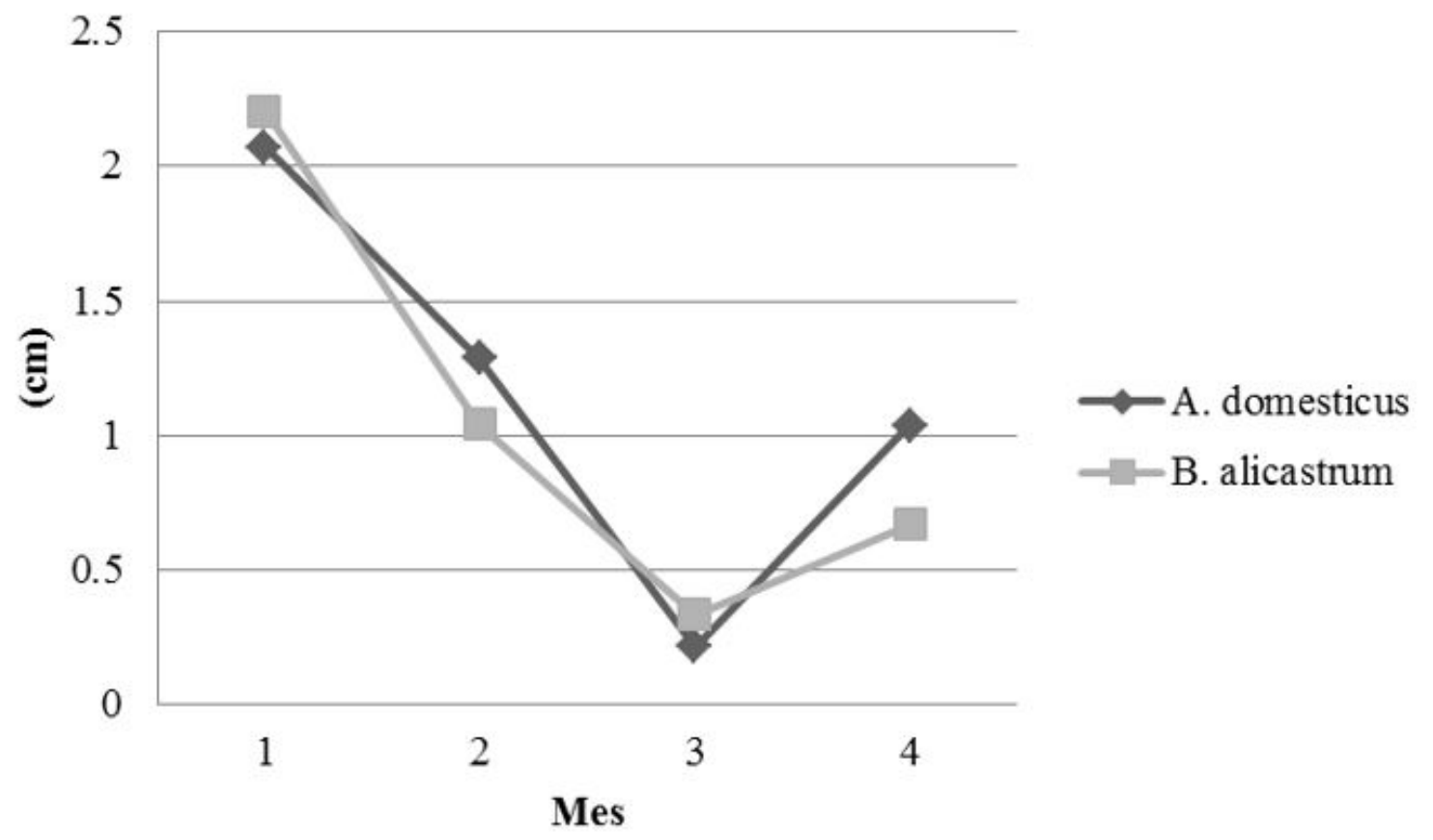

Figura 3. Velocidad de crecimiento en talla

niños de Patulul el 32.1\% presentó desnutrición crónica, además existen otros municipios con niveles más altos de desnutrición que necesitan atención.

Los resultados obtenidos de la caracterización de la frecuencia y tipos de alimentos, demostraron que los niños tienen acceso a diferentes fuentes de proteína (huevo, frijoles y hierbas). Esta dieta no difiere del consumo habitual de las familias a nivel rural en Guatemala (MSPAS, 2012), por lo que el consumo de yogurt enriquecido podría haber influido en la mejora en la ganancia de peso y talla de los niños durante el período en que lo consumieron.

En cuanto a los tratamientos evaluados, se observó una alta aceptación de las características organolépticas de ambos alimentos, lo cual es un importante resultado para lograr que los niños acepten consumir este tipo de productos. Esto facilitará su impacto en las zonas rurales en donde se proyecta el modelo de alimentación. Además de la aceptación se observó que existe mayor preferencia al sabor de yogur con harina de $A$. domesticus, esto puede ser debido al sabor más intenso característico de la harina de $B$. alicastrum el cual según Yates y Ramírez-Sosa (2004) se encontró que presenta sabor diferente al de otras harinas como la de maíz a la que los habitantes de esta zona están acostumbrados.

Los tratamientos de yogur con las diferentes harinas presentaron resultados satisfactorios de ganancias de peso y talla, esto debido a su alto valor nutricional, es por ello que también otros estudios recomiendan su uso en alimentación de escolares (Moreno et al., 2013). El yogur enriquecido con harina de $A$. domesticus presentó los mejores resultados en cuanto peso y talla, tomando en cuenta los datos de Pérez y Sánchez (2012) esta harina aporta una cantidad de nutrientes incluso mayor a la de muchos alimentos comunes en la ingesta diaria: proteínas $60.66 \%$, grasas $31.21 \%$, carbohidratos 4.37 $\%$ y cenizas $3.76 \%$. El aporte energético es aproximadamente $541.1 \mathrm{kcal}$, además de su alto contenido de minerales. Se estima que la utilización de insectos en la alimentación humana será una de las fuentes de mayor riqueza de nutrientes en el futuro (Dossey \& Méndez-Gutiérrez, 2014; Ramos-Elorduy \& Viejo, 2007).

El tratamiento de yogur enriquecido con harina de $B$. alicastrum, presentó menor eficiencia en cuanto a la ganancia de peso y talla en relación al yogur con A. domesticus, sin embargo, si presentó mejor resultado en cuanto a la talla en comparación con el grupo con- 
trol. Estos resultados pueden estar relacionados con una menor digestibilidad de la harina de $B$. alicastrum tal y como lo señalan Arévalo y Bressani (2013).

La velocidad de crecimiento estimada en este estudio para peso y talla, presentó resultados satisfactorios puesto que según los datos de los patrones de crecimiento infantil de la Organización Mundial de la Salud (Multicentre Growth Reference Study Group, 2006), el ritmo de ganancia de peso de niños y niñas entre 4 y 6 años está en el orden de $0.19-0.25 \mathrm{~kg} / \mathrm{mes}$ y la talla en $0.5 \mathrm{~cm} / \mathrm{mes}$ en niños y niñas en el mismo rango de edad. Esto demuestra que las respuestas metabólicas al consumo de yogur con harinas proteicas de origen animal y vegetal en niños en el rango de edad evaluado sobrepasan los niveles normales de velocidad de crecimiento reportados.

Tomando como línea base las guías alimentarias del Ministerio de Salud Pública y Asistencia Social es importante considerar la dieta completa de los niños puesto que al incluir un alimento con alta biodiponibilidad de azúcares y grasas como el yogur sobre la base del aporte adecuado de nutrientes, éste puede provocar algun nivel de sobrepeso en los niños (Farro, Montero, Vergara, \& Ríos-Castillo, 2018). Sin embargo, considerando que los niveles de subnutrición y desbalance alimentario al que están sometidos alrededor de 6 de cada 10 niños en Guatemala, el yogurt es una alternativa viable debido a que reduce la posibilidad de generar intolerancia a la lactosa debido la acción de los microorganismos que contiene (Lactobacillus). Es importante resaltar que la capacidad de generar un ambiente con $\mathrm{pH}$ menor y bacterias no patógenas reduce sustancialmente la persistencia de diarreas en los niños, las cuales se presentan de forma natural por la ingesta y manipulación propia de sus alimentos (Eren, Dinleyici, \& Vandenplas, 2010).

En cuanto al costo de la porción de cada uno de los tratamientos, estos presentaron valores relativamente altos en relación al presupuesto con que se cuenta en las escuelas, tomando como referencia la Ley de Refacción Escolar de Guatemala (Decreto 16-2017), la cual indica que se debe entregar no menos de US\$ 0.52 por beneficiario. El costo mayor del tratamiento con harina de $A$. domesticus, se debe a que existe poca oferta de este producto en el mercado nacional.

La utilización de alimentos funcionales en niños de edad preescolar tiene un impacto positivo en el estado nutricional y futuro desarrollo integral. Es pertinente evaluar la inclusión de este tipo de alimentos en la dieta de la población vulnerable utilizando materias primas existentes en las comunidades.

\section{Agradecimientos}

Esta investigación fue cofinanciada por Digi-Usac-2018, Proyecto: 4.8.63.7.18. Se agradece el apoyo de Miguel Ortiz, por todas las gestiones realizadas para la construcción de la planta de procesamiento de los productos lácteos. De igual forma se agradece a Lesly Alonso de Escuela Tecún Umán de la Finca San Julián, a Navi Ortiz del Centro de Salud de Patulul, Suchitepéquez y a Talia Ovando por el apoyo brindado durante el proyecto.

\section{Referencias}

Apolo-Arévalo, L., \& Lannacone, J. (2015). Crianza del grillo (Acheta domesticus) como fuente alternativa de proteínas para el consumo humano. Scientia, (17), 161-173. doi:10.31381/scientia. v17i17.389

Arévalo, A. I., \& Bressani, R. (2013). Respuesta glicémica de la semilla de Ramón (Brosimum alicastrum) en mujeres de 16 a 25 años de edad, residentes de la ciudad capital. Revista de la Universidad del Valle de Guatemala, 25, 66-71

Barril-Cuadrado, G., Puchulu, M. B., \& Sánchez-Tomero, J. A. (2013). Tablas de ratio fósforo/proteína de alimentos para población española. Utilidad en la enfermedad renal crónica. Nefrología (Madrid), 33(3), 362-371. doi:10.3265/Nefrologia.pre2013.Feb.11918

Britz, M., Aznarez, A., \& Della Santa, A. P. (2017). Desarrollo y validación de ecuaciones para estimar composición corporal en niños de 4 a 6 años de Uruguay. Revista Chilena de Nutrición, 44(1), 63-70. doi:10.4067/S0717-75182017000100009

Carter, C. T. (2015). Chemical and functional properties of Brosimum alicastrum seed powder (Maya nut, Ramón nut) (Tesis de maestria). Universidad Clemson, South California.

Decreto del Congreso de la República, 16-2017. Diario Centro América, No. 24, t. 158. (19 de octubre 2017).

Dossey, A. T., \& Méndez-Gutiérrez, I. R. (2014). Los insectos como una fuente de proteína limpia y sustentable. Entomología Mexicana, 1, 10391044. 
Eren, M., Dinleyici, E. C., \& Vandenplas, Y. (2010). Clinical efficacy comparison of Saccharomyces boulardii and yogurt fluid in acute non-bloody diarrhea in children: A randomized, controlled, open label study. American Journal of Tropical Medicine and Hygiene, 82(3), 488-491. doi:10.4269/ ajtmh.2010.09-0529.

Farro, K., Montero, I., Vergara, E., \& Ríos-Castillo, I. (2018). Elevado consumo de azúcares y grasas en niños de edad preescolar de Panamá: Estudio transversal. Revista Chilena de Nutrición, 45(1), 7-16. doi:10.4067/s0717-75182018000100007

Ghisays, M., Suarez, M., Lastre, G., Fuentes, Y., Orozco, S., Schonewolff, S., \& Verdooren, K. (2018). Estado nutricional de niños menores de cinco años en el caribe colombiano. Ciencia e Innovación en Salud. e62, 1-13. doi:10.17081/innosa.62

Huertas, R. A. (2014). Efecto de la adición de yacon (Smallanthus sonchifolius) en las características fisicoquímicas, microbiológicas, proximales y sensoriales de yogur durante el almacenamiento bajo refrigeración. @limentech, Ciencia y Tecnología Alimentaria, 12(1), 5-14. Doi:10.24054/16927125.v1.n1.2014.423

Lentz, D., Dunning, N. \& Scarborough, V. (2015). Tikal Paleoecology on an Ancient Maya City. New York: Cambridge University Press.

Martínez, K. J. (2014). Desarrollo de la formulación de un atol de harina de maíz enriquecido con harina de semilla de árbol de Ramón (Brosimun alicastrum) evaluación nutricional y diseño del proceso (Tesis de licenciatura). Universidad de San Carlos de Guatemala, Facultad de Ingeniería, Guatemala:

Ministerio de Salud Pública y Asistencia Social. (2012). Guias alimentarias guías alimentarias para Guatemala: Recomendaciones para una alimentación saludable. Guatemala: Autor.

Moreno, L. A., Cervera, P., Ortega, R. M., Díaz, J. J., Baladia, E., ... Salas-Salvadó, J. (2013). Evidencia científica sobre el papel del yogur y otras leches fermentadas en la alimentación saludable de la población española. Nutrición Hospitalaria, 28(6), 2039-2089.

Multicentre Growth Reference Study Group. (2006). WHO Child Growth Standards: Length/heightfor-age, weight-for-age, weight-for-length, weight-for-height and body mass index-for-age: Methods and development. Geneva: World Health Organization.

Nakagaki, B. J., \& Defoliart, G. R. (1991). Comparison of diets for mass-rearing Acheta dornestica (Orthoptera: Gryllidae) as a novelty food and comparison of food conversion efficiency with values reported for livestock. Journal of Economic Entomology 4(3), 891-896.

Nakagaki, B. J., Sunde, M. L., \& Defoliart, G. R. (1986). Protein quality of the house cricket, Acheta domesticus, when fed to broiler chicks. Poultry Science, 66, 1367-1371.

Organización de las Naciones Unidas para la Alimentación y la Agricultura. (2013). Alimentación Escolar y las posibilidades de compra directa de la agricultura familiar. Estudio Nacional de Guatemala. Recuperado de http://www.fao.org/3/ as511s/as511s.pdf

Pérez, R. G., \& Sánchez, R. I. (2012). Elaboración y caracterización de harinas para consumo humano a partir de Achetas domesticus $y$ Periplanetas americanas (Tesis de licenciatura). Universidad Nacional de Trujillo, Facultad de Ingeniería Química, Trujillo, Perú.

Ponce, B. (14 de abril de 2008). ¿Es apropiado el debate vaso de leche escolar versus incaparina? [Mensaje en un blog] Recuperado de http://poncesegura.blogspot.com/2008/04/es-apropiado-el-debate-vaso-de-leche.htl

Ramos-Elorduy, J., \& Viejo, J. L. (2007). Los insectos como alimento humano: Breve ensayo sobre la entomofagia, con especial referencia a México. Boletín Real Sociedad Española de Historia Natural. Sección Biología, 102(1-4), 61-84.

Secretaría de Seguridad Alimentaria y Nutricional. (2015). Plan estratégico de seguridad alimentaria y nutricional PESAN 2016-2020. Recuperado de http://www.sesan.gob.gt/wordpress/wp-content/ uploads/2017/07/PESAN-2016-2020.pdf

Silveira, M., Monereo, S., \& Molina, B. (2003). Alimentos funcionales y nutrición óptima. ¿Cerca o lejos? Revista Española de Salud Pública, 77(3), 317-331.

Sistema de Información Nacional de Seguridad Alimentaria y Nutricional. (2015). Recuperado de http:// www.siinsan.gob.gt/DcxMunicipio 
Victora, C. G., Adair, L., Fall, C., Hallal, P. C., Martore1l, R., Richter, L., \& Sachdev, H. S. (2008). Maternal and child undernutrition 2 maternal and child undernutrition: Consequences for adult health and human capital. Lancet, 371(9609), 340-357. doi:10.1016/S0140-6736(07)61692-4

Yates, S., \& Ramirez-Sosa, C. R. (2004). Ethnobotanical knowledge of Brosimum alicastrum (Moraceae) among urban and rural El Salvadorian adolescents. Economic Botany, 58(1), 72-77. doi:10 .1663/0013-0001(2004)058[0072:EK0BAM]2.0. $\mathrm{CO} ; 2$
Zhong, A. (2017). Product development considerations for a nutrient rich bar using Cricket (Acheta domesticus) protein (Tesis de maestrías). California State University, Long Beach. 